\title{
Politique
}

\section{La démocratie municipale au Québec}

\section{Louise Quesnel}

Numéro 9, hiver 1986

Démocratie et libéralisme

URI : https://id.erudit.org/iderudit/040515ar

DOI : https://doi.org/10.7202/040515ar

Aller au sommaire du numéro

Éditeur(s)

Société québécoise de science politique

ISSN

0711-608X (imprimé)

1918-6584 (numérique)

Découvrir la revue

Citer cet article

Quesnel, L. (1986). La démocratie municipale au Québec. Politique, (9), 61-97. https://doi.org/10.7202/040515ar d'utilisation que vous pouvez consulter en ligne.

https://apropos.erudit.org/fr/usagers/politique-dutilisation/ 


\title{
La démocratie municipale au Québec
}

\author{
Louise Quesnel \\ Université Laval
}

Parmi les façons d'organiser les rapports entre la société civile et l'État, la «démocratie locale» est souvent citée en exemple. Les vertus particulièrement démocratiques du gouvernement municipal semblent acquises et quasi indiscutables, à partir du fait posé que c'est à ce niveau que la distance entre le gouvernement et le gouverné est la plus réduite. Pourtant, il y a belle lurette que la plupart des municipalités ne remplissent plus les conditions qui permettraient au représentant élu de consulter régulièrement tous ses commettants et encore moins à tous les citoyens de se prononcer sur tout ce qui les intéresse. Au niveau local, la «démocratie directe" semble donc révolue et même l'argument traditionnel de l'existence de conditions particulières favorables à la participation des "gouvernés» peut être remis en question.

Pour qu'une analyse dont l'objet touche la gouverne des sociétés soit complète, il faut par ailleurs, faire une place à la société locale et voir comment s'y réalise la gestion des rapports politiques. Fortement encadrés par des règles et des contrôles imposés par le niveau étatique provincial, ces rapports prennent une forme institutionnelle qui est devenue de plus en plus complexe depuis une vingtaine d'années au Québec. Le regard critique que 
nous proposons sur les pratiques démocratiques dans les municipalités québécoises vise à faire le point sur l'état de la «démocratie locale» aujourd'hui, dans le prolongement des mesures récentes qui ont touché la gestion des rapports politiques au niveau municipal, comme par exemple la reconnaissance des partis politiques, le financement des dépenses électorales, l'établissement du suffrage universel, la création d'un niveau régional ou supra-municipal, etc.

Le traitement de faveur que les analystes des gouvernements municipaux ont réservé aux concepts de démocratie, de participation, de décentralisation - à la suite et à l'exemple du discours étatique d'ailleurs qui s'en est inspiré pour appuyer et justifier les grands projets de réorganisation administrative et de réalignement budgétaire - impose que l'on s'arrête d'abord sur ce point, ne seraitce que pour s'intégrer dans le débat théorique actuel. Nous procéderons ensuite, dans une deuxième partie, à l'analyse des différentes modalités de représentation en les abordant selon leur aspect institutionnel et normatif. Les différentes modalités de participation seront ensuite examinées, en troisième partie, encore sous le double éclairage institutionnel et normatif. Nous terminerons avec quelques considérations sur la «crise de la démocratie».

\section{Le débat théorique}

Les bases de la démocratie libérale telle que nous la définissons aujourd'hui, se retrouvent au XIXe siècle alors que, avec le développement de l'économie de marché et de la concurrence, de nouvelles classes sociales revendiquent des droits politiques et l'accès au processus électoral. La démocratie libérale repose alors sur quatre principes de base: 1) la possibilité de désigner les représentants par voie électorale impliquant un choix entre des partis et des candidats; 2) le suffrage élargie (mais non nécessairement 
universel); 3) la liberté de constituer des associations et; 4) la liberté d'opinion et de presse (Macpherson, 1976, 9).

Ces principes soulignent l'importance du processus de désignation des représentants politiques, en même temps qu'ils reconnaissent aussi l'indispensabilité de conditions non strictement électorales, comme la liberté de se regrouper et d'articuler ses points de vue. La démocratie ainsi taillée apparaît comme un mode de gouvernement ou un «système par lequel la majorité des membres d'une société contrôle véritablement ses dirigeants » (Macpherson, 1976, 19). Cette brève définition contient déjà en elle-même quelques-unes des interrogations courantes concernant la démocratie: comment s'établit le contrôle de la société sur les dirigeants? Est-ce un contrôle qui est exercé par la majorité des membres de la société? Quelle place est réservée à la minorité?

Si elle suggère des questions importantes, cette définition en élimine aussi certaines autres. Ainsi, le rapport société-dirigeants, qui est posé comme inévitable, place hors de considération le régime de démocratie directe où les décisions seraient entre les mains des membres de la société, sans intermédiaires élus. Le plus loin qu'il soit possible de se rendre, sans risquer de tomber dans l'utopie, est d'envisager un type de démocratie où les membres de la société, qui sont souvent définis au sens large comme "ceux qui sont affectés par les décisions», se voient reconnaître une certaine forme de participation. L'essentiel de la discussion porte donc, non pas sur la pertinence d'un retour à la démocratie directe, mais sur la forme et l'étendue que doit prendre la participation des membres de la société à la gestion politique:

La démocratie implique essentiellement que les gens affectés par une décision humaine soient pour quelque chose dans cette décision. Il s'ensuit que tout pouvoir décisionnaire doit être légitimé auprès de la collectivité, et que les décisionnaires doivent être tenus pour responsables devant elle. (Mills, 1977, 192). 
Ainsi définie, la démocratie est un idéal dont le degré de réalisation se mesure au niveau de légitimité et de responsabilité des dirigeants à l'égard des membres de la société. La participation, pour être significative, devrait s'insérer dans ce rapport de légitimation et de responsabilisation des dirigeants qui définit essentiellement la démocratie représentative. De plus le processus d'élection des dirigeants et leurs décisions comme gouvernants doivent respecter certaines normes, telles la liberté dans le choix des dirigeants et l'égalité des membres de la société agissant comme électeurs (Lijphart, 1984. 2). En d'autres termes, la démocratie représentative correspond à ce que Bassand et Fragnière, en s'inspirant de G. Burdeau, comme la «démocratie gouvernée».

(...) gouvernement du peuple pour le peuple. Le postulat fondamental de cette conception étant que chaque "citoyen" dispose effectivement du même pouvoir de se faire «représenter» quelle que soit, notamment, sa position dans la structure sociale (Bassand et Fragnière, 1978, 12).

Outre la démocratie directe et la démocratie représentative, d'autres formes de gestion politique sont possibles, comme le socialisme démocratique, le centralisme démocratique et l'autogestion (Hamel et Léonard, 1981, 64-73). Les deux premiers étant plutôt étrangers aux pratiques québécoises, sur lesquelles nous comptons faire porter notre analyse, nous grefferons à l'autogestion le deuxième type de démocratie qui sera retenu, soit la démocratie de participation, qui peut en effet relever en partie de l'autogestion.

Sans remettre entièrement aux concernés l'ensemble des décisions selon le modèle autogestionnaire, la démocratie de participation associe les membres de la société aux décisions et non seulement à l'élection des dirigeants comme le veut la démocratie représentative. Encore en s'inspirant de G. Burdeau, Bassand et Fragnière appellent ainsi la «démocratie gouvernante» le modèle 
qui se rapproche de l'idéal démocratique pur, centré sur le gouvernement du peuple, pour le peuple et par le peuple.

(...) «la démocratie gouvernante» est beaucoup plus fondée sur une tendance à l'autogestion que sur la délégation du pouvoir à des «représentants", même élus (Bassand et Fragnière, 1978, 12-13).

L'expression démocratie de "participation" doit ainsi être comprise en référence à l'accès du "peuple» aux décisions qui le concernent. Mais qui donc est le «peuple» ou qui sont les «membres de la société»? Pour certains il s'agit des usagers ou de ceux qui "participent à l'output» (Godbout, 1983, 38), pour d'autres il s'agit des citoyens résidents (Shearer, 1984, 585), ou pour d'autres il s'agit des classes dominées (Castells, 1982, 67). On souligne cependant parfois que d'autres groupes sociaux participent aux décisions, comme les employés, les agents et les producteurs de services (Vachet, 1985, 168). Il est clair que les rapports politiques au niveau local n'impliquent pas seulement deux grandes catégories d'intervenants, les élus et les citoyens, mais plutôt que la structure du pouvoir est multipolaire. La réflexion sur la démocratie locale peut cependant adopter une problématique centrée sur les rapports entre les dirigeants politiques et l'ensemble des citoyens, sans emprunter la voie de l'analyse des groupes, des élites ou des minorités, bien que de telles analyses soient également pertinentes pour comprendre les rapports politiques. Tout en imposant des limites évidentes, ce dernier cheminement nous semble justifié par la place qu'occupent les citoyens dans les mécanismes institutionnels de représentation et de participation dans les municipalités québécoises, et surtout par l'utilisation de cette problématique dans le discours actuel de la décentralisation et du réaménagement des pouvoirs à l'intérieur de la société québécoise. 


\section{La démocratie de représentation}

Selon le modèle de la démocratie de représentation, les citoyens remettent entre les mains de leurs représentants les décisions qui concernent la collectivité. Comment ces représentants sontils choisis? Les citoyens participent-ils vraiment à ce choix? Qui sont ces représentants Comment exercent-ils cette fonction de représentation des citoyens? En répondant à ces questions, nous tenterons de décrire le fonctionnement plus ou moins démocratique du système de représentation des citoyens dans les municipalités québécoises.

\section{Le choix des dirigeants}

Au-delà de 10,000 élus et élues composent le conseil municipal des quelques 1,600 gouvernements municipaux du Québec ${ }^{1}$. En poste pour quatre ans dans les villes ou pour deux ou trois ans dans un certain nombre de municipalités rurales, ces élus sont choisis à l'automne, à l'occasion d'une élection qui leur est entièrement réservée ${ }^{2}$. Dans les villes, les mandats du maire et des conseillers viennent à échéance en même temps, tandis qu'une partie des élus des municipalités rurales voient leur mandat se

1. Nombre de municipalités au Québec en 1983 selon le nombre d'habitants (Municipalité, déc. 1983, p. 24).
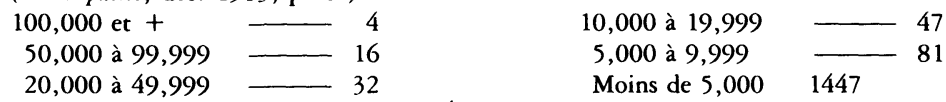

2. Alors qu'en Europe et aux États-Unis par exemple les élections municipales ont lieu en même temps que les législatives, cela n'est jamais le cas ici. Il peut arriver cependant que les élections provinciales suivent de près les municipales comme en 1973, 1976 et 1985. Il n'est pas évident que la séparation des deux scrutins ne nuise pas au premier, soit celui des municipalités puisque l'attention est alors davantage tournée vers les élections provinciales. 
terminer à chaque année ${ }^{3}$. Le maire est élu par l'ensemble des électeurs tandis que chaque conseiller est, en général, choisi dans un district électoral ${ }^{4}$. Il y a donc ici présents certains éléments du régime présidentiel (élection du chef de l'exécutif par l'ensemble des électeurs) et du régime parlementaire (élection des représentants sur la base de districts, à majorité simple des voix).

Ces principales caractéristiques du processus électoral municipal au Québec n'ont pas subi de modifications majeures depuis plusieurs décennies. Certaines pratiques politiques ont cependant été modifiées récemment, à la suite de l'adoption par l'Assemblée Nationale du Québec, d'un ensemble des lois relatives à la démocratie locale ${ }^{5}$. Parmi les points les plus déterminants de ces mesures figurent sans doute la reconnaissance des partis politiques municipaux et le financement des dépenses électorales.

Alors que ces nouvelles règles du jeu existent maintenant depuis cinq ans, leur impact commence à être perceptible ${ }^{6}$, notamment en ce qui concerne le nombre d'élections par scrutin et le nombre de candidats indépendants.

L'élection des dirigeants municipaux a rarement provoqué une affluence de candidats et, selon la tradition, dans la majorité

3. Par exemple, dans une municipalité de 1,000 habitants, le maire et deux conseillers passeront en élection une année tandis que deux autres conseillers attendront à l'année suivante et que les deux derniers termineront leur mandat dans deux ans. Selon ce principe du renouvellement partiel des mandats, il n'y a donc jamais d'élection générale dans les petites municipalités, sauf en cas de démission simultanée de tous les membres du conseil.

4. Dans certaines municipalités rurales, il n'y a pas de districts électoraux et les conseillers sont élus par l'ensemble des électeurs selon le principe des sièges. Un électeur votera ainsi autant de fois qu'il y aura de sièges à remplir et une fois de plus s'il y a également élection à la mairie.

5. Loi concernant les élections de 1978 dans certaines municipalités et modifiant la Loi des Cités et villes, adoptée le 22 juin 1978 (Loi 44); Loi modifiant certaines dispositions législatives concernant la démocratie et la rémunération des élus dans les municipalités, adoptée le 18 juin 1980 (Loi 105).

6. En 1985 , l'observation est d'autant plus intéressante que nous voyons la deuxième génération des élections municipales soumises à ces nouvelles règles, quatre ans après la première application en 1981 . 
des cas, le candidat unique était déclaré élu par acclamation le jour même des mises en candidature. La situation a peu changé récemment (tableau 1 et 2 ) puisque plus des deux-tiers des postes de maires et de conseillers sont comblés sans opposition ${ }^{7}$. La tenue d'un scrutin au niveau municipal constitue une exception si l'on considère l'ensemble des municipalités québécoises. Cependant, le grand nombre de municipalités et leur diversité impose un raffinement à l'analyse.

À la suite de la loi qui distingue les villes de 20,000 habitants et plus en leur imposant des règles spécifiques en ce qui concerne le découpage des districts électoraux, le financement des dépenses électorales et la déclaration d'intérêt des nouveaux élus, l'observaion des pratiques indique une compétition plus élevée dans ces villes où les élections par acclamation figurent à titre d'exception. Ainsi, la ville de Québec n'a connu entre 1965 et 1985 qu'une élection

TABLEAU 1

Candidats élus au poste de maire par acclamation, selon le sexe, pour toutes les municipalités du Québec, 1981 à 1985

\begin{tabular}{|ccccc|}
\hline & Hommes & Femmes & Total & $\begin{array}{c}\% \\
\text { par rapport au total } \\
\text { de postes à pourvoir }\end{array}$ \\
\hline 1981 & 574 & 13 & 587 & 67,2 \\
1982 & 107 & 4 & 111 & 51,6 \\
1983 & 600 & 20 & 620 & 68,7 \\
1984 & 79 & 2 & 81 & 60,0 \\
1985 & ND & ND & 657 & 71,0 \\
\hline
\end{tabular}

Source: 1981 à 1984: Ministère des affaires municipales, Direction de la recherche et des politiques, 1985.

1985: Le Soleil, 3 novembre 1985.

7. En 1982, presque la moitié des postes de maires qui venaient à échéance ont été comblés suite à un scrutin (tableau 1). Cette situation exceptionnellement active risque de se produire en 1986 alors que les mêmes municipalités seront en élection. La liste des villes de cette catégorie peut être consultée dans Municipalité, août-septembre 1982 , p. 15. 
TABLEAU 2

Candidats élus aux postes de conseillers par acclamation selon le sexe, pour toutes les municipalités du Québec 1981 à 1985.

\begin{tabular}{|ccccc|}
\hline & Homme & Femme & Total & $\begin{array}{c}\% \\
\text { par rapport au total } \\
\text { de postes à pourvoir }\end{array}$ \\
\hline 1981 & 2171 & 169 & 2340 & 68,5 \\
1982 & 2127 & 189 & 2316 & 70,2 \\
1983 & 2236 & 225 & 2461 & 68,6 \\
1984 & 1799 & 257 & 2056 & 78,1 \\
1985 & ND & ND & 2663 & 73,2 \\
\hline
\end{tabular}

Source: 1981 à 1984: Ministère des affaires municipales, Direction de la recherche et des politiques, 1985.

1985: Le Soleil, 3 novembre 1985.

par acclamation, en 1973; et Montréal, ville de Laval et Longueuil n'en ont connu aucune au cours de la même période.

De plus, les dispositions juridiques visant à reconnaître officiellement les partis politiques sur la scène municipale ${ }^{8}$, adoptées en 1979 ont donné lieu à la formation de partis locaux dans une trentaine de villes de 20,000 habitants et plus entre 1982 et 1985 (tableau 3). Si le nombre de villes devenues officiellement partisanes semble stable, par contre la présence de deux et même de trois partis est plus fréquente en 1985 qu'en 1982, ce qui laisse croire en l'implantation d'un véritable système de parti, tout au moins en période électorale, dans une vingtaine de villes québécoises.

L'expérience de quelques années au sein d'un conseil municipal multipartisan a mené certains conseillers siégeant auparavant comme «indépendants» à se rallier à un parti, comme ce fut le cas à Sainte-Foy où aucun candidat indépendant ne persiste aux élections

8. Il s'agit de partis municipaux, sans affiliation officielle avec les partis politiques nationaux (voir à ce sujet $\mathbf{L}$. Quesnel-Ouellet, 1982). Cette pratique est très différente des expériences américaines, européennes ou scandinaves où les partis nationaux sont présents aux élections municipales et où les partis strictement locaux sont peu nombreux et très marginaux. 
de 1985, les quarante-deux candidats et candidates aux quatorze postes de conseillers étant tous et toutes inscrits sous la bannière de l'un des trois partis en lice. De l'avis de ces «ex-indépendants", le parti leur offre des avantages appréciables au niveau de l'organisation de la campagne électorale, tout comme il facilite leur intégration à l'appareil politique municipal (comités et commissions) dont sont souvent exclus les conseillers indépendants au sein d'un conseiller partisan.

TABLEAU 3

Présence du système de partis dans les municipalités où il y a des partis politiques municipaux autorisés

1982 et 1985

\begin{tabular}{|lcc|}
\hline & 1982 & $1985^{(1)}$ \\
\hline $\begin{array}{l}\text { Municipalités où un seul parti a } \\
\text { été autorisé }\end{array}$ & 15 & 8 \\
$\begin{array}{l}\text { Municipalités avec système de partis } \\
\text { Total }\end{array}$ & 15 & \\
2 partis autorisés & 12 & 21 \\
3 partis ou + autorisés & $3^{(2)}$ & 14 \\
\hline
\end{tabular}

(1): Entre 1978 et 1985, vingt-trois partis ont été reconnus officiellement puis démantelés. Ils ne sont pas compilés ici.

(2): Laval, Montréal et St-Léonard.

(3): Montréal, St-Léonard, Beauport, St-Hubert, Lava, Ste-Foy et Verdun.

Source: Colloque "Les principaux aspects de la réforme électorale au niveau municipal", Statistiques et analyse relatives à l'application des chapitres II, III, et VII de la loi sur les élections dans certaines municipalités. Directeur général de la représentation électorale, 1982. Direction générale des élections, Liste des partis politiques municipaux accrédités de 1982 à 1985 .

Aux élections municipales, le nombre de candidats et candidates et la fréquence des affrontements électoraux n'ont donc pas varié de façon significative au cours des dernières années dans l'ensemble des municipalités. Le remaniement de la carte électorale dans les grandes villes, à la faveur de districts électoraux plus nombreux et plus petits, a toutefois contribué à augmenter le nombre de 
postes de conseillers à pourvoir et, conséquemment, le nombre de candidats et candidates. Par exemple celui-ci est passé de 8 à 42 à Sainte-Foy entre 1969 et 1985 , et de 29 à 45 à Québec de 1969 à 1985 (tableau 4). Les ressources mises à la disposition des candidats et candidates ont augmenté avec le remboursement de la moitié des dépenses électorales de ceux et celles qui ont obtenu plus de vingt pourcent des voix exprimées. Ces avantages s'accompagnent cependant de contrôles étatiques sur le type et la somme totale des dépenses admissibles de même que sur les contributions des sympathisants. Les pratiques électorales des candidats et candidates ont donc connu quelques modifications importantes, surtout dans les centres urbains. Dans la plupart des municipalités du Québec, celles qui comptent moins de 1,000 habitants, la politique locale s'active surtout en période pré-électorale, en vue de dénicher le candidat ou la candidate qui soit agréable à tous et qui évite la tenue d'une élection.

TABLEAU 4

Nombre de candidats et nombre de districts électoraux aux postes de conseiller municipal, Sainte-Foy et Québec, 1969 à 1985.

\begin{tabular}{|c|ccc|cccc|}
\hline & \multicolumn{3}{|c|}{ Sainte-Foy } & \multicolumn{3}{c|}{ Québec } \\
\hline & $\begin{array}{c}\text { Candi- } \\
\text { dats }\end{array}$ & $\begin{array}{c}\text { Districts } \\
\text { électoraux }\end{array}$ & $\begin{array}{c}\text { Électeurs } \\
\text { inscrits }\end{array}$ & $\begin{array}{c}\text { Candi- } \\
\text { dats }\end{array}$ & $\begin{array}{c}\text { Districts } \\
\text { électoraux }\end{array}$ & $\begin{array}{c}\text { Électeurs } \\
\text { inscrits }\end{array}$ \\
\hline 1969 & 8 & 6 & 27,296 & 29 & $3^{(1)}$ & 103,871 \\
1973 & 11 & 7 & 41,637 & $(2)$ & $7^{(3)}$ & ND \\
1977 & 14 & 7 & 49,959 & 53 & 7 & 118,995 \\
1981 & 34 & 14 & 49,157 & 48 & 21 & 116,083 \\
1985 & 42 & 14 & 47,688 & 45 & 21 & 119,651 \\
\hline
\end{tabular}

(1): En 1969, la ville compte 3 grands districts, Champlain, St-Roch, et Limoilou, avec 4 sièges chacun.

(2): En 1973, le maire sortant est réélu par acclamation ainsi que 14 des 16 conseillers.

(3): Trois districts pour la ville selon les anciennes limites, soit Champlain, St-Roch/ St-Sauveur et Limoilou, comptent chacun quatre sièges. Quatre districts, Les Saules, Duberger, Neufchatel et Charlemagne-ouest, ont un siège chacun.

Source: Pour Sainte-Foy: C. Forand (1985)

Pour Québec: Rapport du Président des élections de la ville de Québec. 
Le processus électoral s'insère dans la problématique de la démocratie locale en tant que mode d'organisation des rapports entre les citoyens et leurs représentants élus. Comment les citoyens se situent-ils dans ce processus?

\section{La pratique du choix électoral}

Les élections constituent le principal instrument formel d'intervention des citoyens au niveau municipal, en ce qu'elles mobilisent le plus grand nombre de personnes ${ }^{9}$. Selon les théoriciens de la démocratie libérale, les électeurs libres et égaux remplissent alors un rôle primordial, inspiré par une logique qui s'appuie sur une information variée, et posent un choix entre différents candidats et plusieurs options politiques (Lijphart, 1984, 2) ${ }^{10}$.

Au Québec, ce choix est restreint au tiers des municipalités, puisque les autres deux tiers ne connaissent pas la «fébrilité» des campagnes électorales. Dans ce dernier cas, les dirigeants municipaux sortant de charge sont réélus par acclamation ou, s'ils ne désirent plus occuper de postes au niveau municipal, ils voient à se trouver un successeur qui rallie une majorité avant même que les élections ne soient tenues. Le "choix» électoral est donc pratiquement réservé aux citoyens des villes et de quelques centaines de municipalités rurales.

On a beaucoup parlé du faible intérêt des électeurs et du niveau de participation plus bas aux élections municipales que lors des scrutins provinciaux et fédéraux (Bernard, 1976, 151). Dans l'ensemble des municipalités québécoises, le nombre de

9. Dans les grandes villes, le nombre de votants pour les candidats au poste de maire dépasse même le nombre d'électeurs inscrits dans les plus grandes circonscriptions électorales provinciales, ce qui permet d'affirmer par exemple que le maire de Montréal, Jean Drapeau, est l'élu québécois dont les appuis électoraux sont les plus nombreux $(83,908$ voix en 1982 , par exemple). (1977).

10. Cet idéal démocratique est sérieusement attaqué dans Braud (1980) et Kolm 
votants représente en effet autour de cinquante pourcent des électeurs inscrits pour l'élection au poste de maire (tableau 5), tandis qu'un pourcentage légèrement inférieur est constaté aux postes de conseillers, de 1981 à 1984 (tableau 6). Une différence importante apparaît cependant lorsque les rnunicipalités sont distinguées selon leur dimension et même lorsqu'à l'intérieur d'une même ville, les districts électoraux sont comparés. Dans la catégorie des petites municipalités de moins de 10,000 habitants (tableau 7), nous constatons que les villes (municipalités relevant de la loi des cités et villes, LCV) connaissent une participation électorale substantiellement plus élevée que les villages et les paroisses (municipalités relevant du Code municipal, CM). De plus, la participation électorale est sensiblement plus élevée dans les municipalités comptant moins de 1,000 habitants que dans celles qui en comptent entre cinq et dix milles. Enfin, dans les petites villes de 3,000 à 10,000 habitants, la participation électorale a augmenté de plus de dix points.

En ce qui concerne les plus grandes villes, des fluctuations importantes sont manifestes d'une élection à l'autre (tableau 8). Les explications évoquées concernent les candidats, les plate-formes des organisations partisanes et les caractéristiques des électeurs (par exemple Beauregard, 1984; Léveillée, 1978). Comme dans le cas des petites municipalités, il n'est pas évident que la participation électorale connaisse partout une tendance à la hausse. Il semble cependant que certaines conjonctures provoquent un soubresaut de la part de l'électorat, comme la préparation de l'Exposition universelle de Montréal en 1967 qui a valu un appui généralisé au maire sortant en 1966 et conséquemment un désintérêt de cette élection sans enjeu, ou comme la «crise d'octobre» en 1979 qui a, au contraire, suscité un intérêt spécifique, ou comme la présence de trois candidats forts qui se sont affrontés pour succéder à un maire à sa retraite à Sainte-Foy en 1985 . 


\section{TABLEAU 5}

Taux moyen de participation électorale, élection à la mairie, municipalités relevant du Code municipal et municipalités relevant de la Loi des cités et villes, 1981 à 1984.

\begin{tabular}{|c|c|c|c|c|c|}
\hline & $\begin{array}{l}\text { Municipali } \\
\text { relevant du } \\
\text { municipa }\end{array}$ & Code & $\begin{array}{r}\text { Municip } \\
\text { relevant de la } \\
\text { et vil }\end{array}$ & les cités & $\begin{array}{c}\text { Total des } \\
\text { Municipalités }\end{array}$ \\
\hline & $\begin{array}{l}\text { Nombre de } \\
\text { Municipalités }\end{array}$ & Taux & $\begin{array}{l}\text { Nombre de } \\
\text { Municipalités }\end{array}$ & Taux & Taux \\
\hline 1981 & 249 & 50,8 & 37 & 48,0 & 49,0 \\
\hline 1982 & 47 & 52,1 & 57 & 52,6 & 52,6 \\
\hline 1983 & 239 & 58,1 & 44 & 58,8 & 58,5 \\
\hline 1984 & 45 & 49,4 & 9 & 54,4 & 52,8 \\
\hline
\end{tabular}

Source: Ministère des affaires municipales, Direction de la recherche et des politiques, 1985.

\section{TABLEAU 6}

Taux moyen de participation électorale, élection au poste de conseiller, municipalités relevant du Code municipal et municipalités relevant de la Loi des cités et villes, 1981-1984.

\begin{tabular}{|c|c|c|c|c|c|}
\hline & \multicolumn{2}{|c|}{$\begin{array}{c}\text { Municipalités } \\
\text { relevant du Code municipal }\end{array}$} & \multicolumn{2}{|c|}{$\begin{array}{l}\text { Municipalités } \\
\text { relevant de la Loi des cités } \\
\text { et villes }\end{array}$} & \multirow{2}{*}{$\begin{array}{c}\begin{array}{c}\text { Total des } \\
\text { Municipalités }\end{array} \\
\text { Taux }\end{array}$} \\
\hline & $\begin{array}{l}\text { Nombre de } \\
\text { Municipalités }\end{array}$ & Taux & $\begin{array}{l}\text { Nombre de } \\
\text { Municipalités }\end{array}$ & Taux & \\
\hline 1981 & 432 & 44,3 & 59 & 48,6 & 46,1 \\
\hline 1982 & 316 & 42,3 & 87 & 51,4 & 49,1 \\
\hline 1983 & 402 & 51,8 & 66 & 53,4 & 52,5 \\
\hline 1984 & 274 & 41,8 & 12 & 55,0 & 46,0 \\
\hline
\end{tabular}

Source: Ministère des affaires municipales, Direction de la recherche et des politiques, 1985. 
TABLEAU 7

Taux moyen de participation électorale, élection à la mairie, municipalités de moins de 10,000 habitants, relevant du Code municipal et de la Loi des cités et villes,

1981-1984.

\begin{tabular}{|llllllllll|}
\hline & \multicolumn{2}{c}{1981} & \multicolumn{2}{c}{1982} & & 1983 & & 1984 \\
\hline $\begin{array}{c}\text { Taille des } \\
\text { Municipalités }\end{array}$ & $\begin{array}{c}\text { Code } \\
\text { Mun. }\end{array}$ & LCV & $\begin{array}{c}\text { Code } \\
\text { Mun. }\end{array}$ & LCV & $\begin{array}{c}\text { Code } \\
\text { Mun. }\end{array}$ & LCV & $\begin{array}{c}\text { Code } \\
\text { Mun. }\end{array}$ & LCV \\
\hline Moins de 999 & 56,5 & 65,6 & 63,2 & 73,5 & 56,7 & - & 51,6 & - \\
1000 à 2999 & 52,7 & 69,2 & 55,5 & 70,5 & 61,4 & 66,2 & 47,7 & - \\
3000 à 4999 & 45,2 & 57,1 & 53,0 & 60,7 & 55,3 & 64,3 & 50,4 & 73,2 \\
5000 à 9999 & 43,4 & 48,6 & 28,6 & 58,2 & 52,8 & 64,7 & 53,6 & 65,3 \\
Nombre de & & & & & & & & \\
Municipalités & 249 & 22 & 47 & 31 & 239 & 23 & 45 & 3 \\
\hline
\end{tabular}

Source: Ministère des affaires municipales, Direction de la recherche et des politiques, 1985.

TABLEAU 8

Pourcentage de votants par rapport aux électeurs inscrits aux élections municipales, Montréal, Québec, Sainte-Foy, 1965 à 1985.

\begin{tabular}{|cccc|}
\hline & Montréal & Québec & Sainte-Foy \\
\hline 1965 & - & $61 \%$ & ND \\
1966 & $33 \%$ & - & - \\
1969 & - & $61 \%$ & $30 \%$ \\
1970 & $53 \%$ & - & - \\
1973 & - & - & $45 \%$ \\
1974 & $38 \%$ & $46 \%$ & - \\
1977 & - & - & $33 \%$ \\
1978 & $54 \%$ & - & - \\
1981 & - & $53 \%$ & - \\
1982 & - & $53 \%$ & $59 \%$ \\
1985 & & &
\end{tabular}

(1) Le maire et 14 des 16 conseillers municipaux sont élus par acclamation.

La pertinence des classes sociales pour analyser la participation électorale est aussi visible, particulièrement lorsque celle-ci est observée au niveau des districts électoraux. Ce niveau permet en 
effet de rejoindre les groupes sociaux en écartant l'effet d'homogénéité que crée l'analyse plus globale, particulièrement dans les villes dont la composition sociale n'est pas homogène. Par exemple, à Sainte-Foy en 1981, la participation électorale était de $21 \%$ dans le district électoral universitaire de Laval, et de $50 \%$ dans le district plus résidentiel et plus bourgeois de Laurier (tableau 9).

\section{TABLEAU 9}

Participation électorale, pourcentage de logements occupés par un locataire et valeur moyenne du logement, selon le district électoral, ville de Sainte-Foy, élections municipales, 1981.

\begin{tabular}{|c|c|c|c|}
\hline $\begin{array}{l}\text { District } \\
\text { électoral }\end{array}$ & $\begin{array}{l}\text { Participation } \\
\text { électorale }\end{array}$ & $\begin{array}{c}\% \text { de } \\
\text { locataires } \\
(1981)\end{array}$ & $\begin{array}{c}\text { Valeur moyenne } \\
\text { du logement } \\
\text { (1981) }\end{array}$ \\
\hline St-Thomas & 41 & $\begin{array}{l}68 \\
96\end{array}$ & $\$ 67063$. \\
\hline St-Jean-Baptiste & 45 & 53 & $\$ 68201$. \\
\hline St-Denis & 40 & 75 & $\$ 69754$ \\
\hline St-Yves & 50 & 20 & $\$ 75908$ \\
\hline Laurier & 50 & 44 & $\$ 80970$ \\
\hline $\begin{array}{l}\text { St-Mathieu } \\
\text { Notre-Dame }\end{array}$ & $\begin{array}{l}46 \\
44\end{array}$ & $\begin{array}{l}65 \\
65\end{array}$ & $\$ 58362$. \\
\hline Ste-Geneviève & 46 & 52 & \multirow{2}{*}{$\begin{array}{c}\$ 52344 . \\
(1)\end{array}$} \\
\hline St-Benoît & 54 & 40 & \\
\hline Neilson & 36 & 74 & \multirow{2}{*}{$\begin{array}{c}\$ 69184 . \\
\text { (1) }\end{array}$} \\
\hline Pointe Ste-Foy & 37 & 64 & \\
\hline Champigny & 44 & 20 & \multirow{3}{*}{$\$ 45030$} \\
\hline Chauveau & 44 & 18 & \\
\hline Total ville & $43 \%$ & $57 \%$ & \\
\hline
\end{tabular}

(1) Les limites des secteurs de recensement utilisés par Statistique Canada sont différentes de celles des districts électoraux. Le district St-Benoît partage avec Pointe Ste-Foy trois secteurs de recensements où la valeur moyenne du logement est respectivement de $\$ 72,739$., $\$ 68,795$. et $\$ 66,910$.

Source: Rapport officiel du Président des élections, 1981.

Statistique Canada, Recensement de 1981. 
Le «choix» électoral, celui de voter d'abord, et celui d'opter pour un candidat ensuite, est donc influencé par de nombreux facteurs dont l'effet conjugué, et difficile à disséquer, se traduit par l'élection d'un maire et d'un conseiller. Ces derniers, comme candidats, tentent de différentes façons de se gagner les faveurs des électeurs. Dans le cas des petites municipalités, les électeurs sont courtisés par des candidats «indépendants», c'est-à-dire non identifiés à un parti politique municipal ou à une équipe. Dans cette dernière situation, qui est encore la plus fréquente, les candidats au poste de maire et aux postes de conseillers se présentent seul, faisant valoir leur propre personnalité et leur disponibilité à gérer efficacement le village ou la paroisse.

Le principe de «l'équipe» gagne cependant en popularité depuis une dizaine d'années. Ainsi, un candidat à la mairie (souvent en fin de mandat) regroupe-t-il autour de lui quelques conseillers également sortant de charge, y ajoute une ou deux nouvelles figures et présente ainsi son «équipe». Cette formule, intermédiaire entre la candidature isolée de «l'indépendant» et celle plus encadrée du partisan, comporte certains atouts: elle permet de partager des tâches d'organisation électorale, elle assure les nouveaux candidats de l'expérience et de l'appui des anciens, tout en ne provoquant pas la réaction de méfiance que la formule carrément partisane entraine encore chez bon nombre d'électeurs et de dirigeants municipaux ${ }^{11}$.

Du point de vue du choix électoral, la formule de l'équipe demeure toutefois insatisfaisante puisqu'elle incite à la formulation d'options politiques et qu'elle est plus axée sur une fonction de

11. L'opposition à l'entrée des partis politiques sur la scène municipale vient du mouvement réformiste du début du XXe siècle dont l'influence considérable se fait encore sentir, notamment à propos du caractère essentiellement administratif des décisions à prendre au niveau municipal (Tindal et Tindal, 1984, 150-152, Magnusson et Sancton, $1983,30)$. 
regroupement de ressources que sur une fonction d'élaboration de programme ou même de plate-forme électorale.

Encore toute neuve au Québec, la formule partisane ressemble à celle de l'équipe et se rapproche prudemment de celle du régime des partis politiques provinciaux ou fédéraux. À l'instar de Montréal et Québec où des organisations partisanes existent depuis respectivement 1954 et $1962^{12}$, et où des partis d'opposition se sont constitués en 1974 et en $1977^{13}$, les électeurs de plusieurs dizaines de ville de 20,000 habitants et plus sont confrontés à un système de partis municipaux. Surtout actifs en période électorale, ces partis sont encore aujourd'hui ${ }^{14}$ souvent menés par un candidat à la mairie qui fait figure d'âme dirigeante, et dont le leadership va même jusqu'à lui réserver le privilège du choix des candidats du parti aux postes de conseiller.

Préoccupés de questions reliées à l'environnement, à l'habitation, à la fiscalité municipale, ou à la démocratie locale, les partis dans l'ensemble ne sont pas fondamentalement opposés du point de vue idéologique. N'étant pas, en général, liés par un programme partisan mais plutôt par une plate-forme électorale, ils adaptent leurs thèmes à l'actualité, en ayant comme toile de fond quelques grands principes relevant souvent davantage de la vertu que l'engagement politique, comme par exemple la réduction du compte de taxes, l'information des citoyens et, dans le cas des candidats demandant un renouvellement de mandat, la qualité du travail accompli. Force est de constater que les partis municipaux ressemblent en cela à leurs homologues provinciaux et fédéraux

12. À Montréal, la ligue d'action civique est fondée en 1954. À Québec, le Progrès Civique de Québec est fondé en 1962.

13. Le Rassemblement des citoyens de Montréal en 1974 et le Rassemblement populaire de Québec en 1977.

14. Cette situation était présentée comme une caractéristique des «alphabet parties» qui sont apparus dans quelques villes canadiennes au cours des années soixante (Joyce et Hossé, 1970). 
et que les débats électoraux ne connaissent pas, au niveau municipal, un degré de raffinement plus élevé. Nous constatons cependant que là où plusieurs partis s'affrontent, les critiques des uns sont reprises par les autres et un parti qui n'aurait pas, de lui-même, choisi d'aborder la question de l'habitation pour les personnes âgées, par exemple, est forcé de réagir aux mesures proposées par son concurrent sur ce point. Cette surenchère des thèmes entraîne une homogénéisation relative des plate-formes électorales tout en amenant les candidats à considérer un ensemble très vaste de sujets. Les électeurs y sont probablement gagnants, même si la situation n'est pas toujours claire quand vient le jour du scrutin.

Les candidats ont, eux aussi, de la difficulté à s'y retrouver. Quelques-uns vont «s'offrir» à plus d'une formation partisane, d'autres vont passer d'un parti à un autre. Cette situation qui se constate également aux niveaux provincial et fédéral, est cependant plus fréquente et peut être plus acceptée au niveau municipal où la tradition partisane est, à toutes fins pratiques, inexistante.

Parmi les quatre-vingt-sept partis municipaux dont la formation a été autorisée depuis 1978, il en est quelques-uns qui se démarquent du portrait général qui vient d'être tracé. Il s'agit de partis qui regroupent un nombre de membres important (quelques centaines), qui ont un programme dûment adopté en congrès, dont les candidats sont choisis lors de convention dans les districts, et dont les élus adoptent régulièrement au conseil municipal, une ligne de parti qui se traduit entre autres formes, par une position commune à l'occasion des votes. Présents sur la scène politique locale au cours des trois dernières élections municipales, ces partis y ont agi comme principaux porte-parole de la minorité des électeurs. Le scrutin majoritaire à un tour ne leur a cependant pas accordé un nombre de sièges équivalent au poids relatif que leur a réservé le vote populaire (tableau 10). La majorité fut donc sur-représentée au conseil municipal de ces villes où le même parti domine largement depuis au-delà de vingt ans. (tableau 11). 
Puisque selon le mode de scrutin en vigueur dans les municipalités, les électeurs exercent deux votes, l'un au poste de maire et l'autre au poste de conseiller du district où ils demeurent, ces deux votes vont-ils aux candidats du même parti? Dans quelques villes, par exemple Longueuil en 1978, et Sainte-Foy en 1985, le maire élu a dû faire face à un conseil où les représentants aux postes de conseiller de son propre parti étaient minoritaires. Cela signifie que bon nombre d'électeurs ont voté à la mairie et au poste de conseiller pour des candidats de partis différents. Ce comportement apparamment incohérent, s'explique par l'importance

\section{TABLEAU 10}

Pourcentage des voix et nombre d'élus, principal parti d'opposition à Montréal et à Québec, au cours des trois dernières élections.

\begin{tabular}{|lcc|cc|}
\hline & $\begin{array}{c}\text { Rassemblement des citoyens et } \\
\text { citoyennes de Montréal }\end{array}$ & \multicolumn{2}{c|}{$\begin{array}{c}\text { Rassemblement populaire } \\
\text { de Québec }\end{array}$} \\
\hline & $\%$ des voix & Nombre d'élus & $\%$ des voix & Nombre d'élus \\
\hline 1974 & $39 \%$ & 18 sur 55 & - & - \\
$1977^{(1)}$ & - & - & $24 \%$ & - \\
$1978^{(2)}$ & $18 \%$ & 1 sur 54 & - & 4 sur 21 \\
1981 & - & - & - & - \\
$1982^{(3)}$ & $36 \%$ & 15 sur 57 & $40 \%$ & 4 sur 21 \\
1985 & - & - & & \\
\hline
\end{tabular}

(1) En 1977, le Parti municipal obtient $13 \%$ des voix à Québec et ne fait élire aucun conseiller. Le parti majoritaire, le Progrès civique de Québec, remporte tous les sièges de conseillers avec $58 \%$ des voix.

(2) En 1978, à Montréal, le GAM fait élire un seul représentant tout en recueillant $26 \%$ des voix. Le RCM se place en troisième position.

(3) En 1982, le Groupe d'action municipale recueille $15 \%$ des voix et fait élire trois conseillers qui composeront l'opposition avec le RCM.

Source: Rapports du Président des élections de Montréal, 1974, 1978, 1982. Rapports du Président des élections de Québec, 1977 et 1981. Pour 1985, il s'agit des résultats non officiels tels que rendus publics le 17 novembre 1985 . 


\section{TABLEAU 11}

Appui électoral accordé au maire et à son parti, Montréal et Québec, élections municipales, 1969 à 1985.

\begin{tabular}{|c|ccc|ccc|}
\hline & \multicolumn{3}{|c|}{ Montréal } & \multicolumn{3}{c|}{ Québec } \\
\hline & $\begin{array}{c}\text { E des voix } \\
\text { Électeurs } \\
\text { inscrits }\end{array}$ & $\begin{array}{c}\text { Siège de } \\
\text { au maire } \\
\text { conseillers } \\
\text { au PCM }\end{array}$ & $\begin{array}{c}\text { Électeurs } \\
\text { inscrits }\end{array}$ & $\begin{array}{c}\text { \% des voix } \\
\text { accordées } \\
\text { au maire }\end{array}$ & $\begin{array}{c}\text { Siège de } \\
\text { conseillers } \\
\text { au PCQ }\end{array}$ \\
\hline 1969 & - & - & - & 103,871 & $64 \%$ & 12 sur 12 \\
1970 & 698,753 & $92 \%$ & 52 sur 52 & - & - & - \\
1973 & - & - & - & ND & Acclamation & 16 sur 16 \\
1974 & 687,369 & $55 \%$ & 36 sur 55 & - & - & - \\
1977 & - & - & - & 118,995 & $58 \%$ & 16 sur 16 \\
1981 & $-665,276$ & $60 \%$ & 52 sur 54 & - & - & - \\
1982 & 707,000 & $49 \%$ & 39 sur 57 & - & - & 116,083 \\
1985 & - & - & - & 119,651 & $58 \%$ & 17 sur 21 \\
\hline
\end{tabular}

persistante du candidat comme individu et non comme partisan et par la faiblesse de la tradition partisane dans les municipalités ${ }^{15}$.

La tâche des partis minoritaires n'est donc pas facile puisqu'ils doivent se tailler une place et modifier des pratiques politicoadministratives bien établies. Ils ont cependant obtenu de l'Assemblée Nationale du Québec que soit votée en 1984 une loi obligeant les trois plus grandes villes, Montréal, Laval et Québec, à leur accorder des services de secrétariat et un budget de recherche. Ils pourront ainsi remplir avec une efficacité accrue leur fonction de représentation, comme leurs collègues majoritaires au conseil qui bénéficient à l'exclusivité, de l'apport des employés municipaux

15. La position difficile dans laquelle se trouve alors le maire fait ressortir, selon certains, la nécessité d'une réforme du mode de désignation du maire. En conformité avec les principes du régime parlementaire, le maire devrait plutôt être élu comme conseiller puis comme chef du gouvernement si son parti obtient la majorité des sièges. Les dirigeants politiques municipaux, notamment les maires actuels, ne favorisent pas tellement cette mesure qui est, par ailleurs, proposée par le RPQ à Québec. 
et même d'un cabinet politique dans le cas du président du comité exécutif.

\section{Le profil des dirigeants municipaux}

Les élus municipaux sont désignés pour représenter les citoyens et prendre, à leur place, une part active et directe à la gouverne de la société locale. La démocratie de représentation est souvent analysée à l'aide du concept de représentativité selon lequel les dirigeants qui sont proches des citoyens seraient plus aptes à traduire leurs volontés. Les points de vue sur la façon la plus appropriée d'être "proches des citoyens" penchent soit du côté d'une interprétation subjective, soit du côté d'une interprétation objective. La première, couramment utilisée par les dirigeants eux-mêmes, se base sur la capacité de compréhension des intérêts, sur la connaissance et l'expérience des dirigeants. La seconde, plutôt privilégiée, comme ce sera le cas ici, par les analystes, insiste sur les caractéristiques socio-économiques des candidats et compare le profil des élus à celui des citoyens. Les deux critères peuvent être considérés comme des révélateurs de la légitimité dont sont investis les élus par rapport aux citoyens.

Suite à une enquête réalisée en 1979 auprès d'un échantillon de maires et de conseillers municipaux ${ }^{16}$, on apprend qu'à ce moment, l'âge moyen des maires est de cinquante ans, que $95 \%$ des maires et des conseillers sont propriétaires du logement où ils résident, et que $96 \%$ sont de sexe masculin. Ce profil a-t-il subi quelques modifications au cours des dernières années?

Il est largement évident que les municipalités sont encore «un monde d'hommes» (Tardy, 1982): les mairesses ne comptent même pas pour $5 \%$ du total des candidats élus au poste de maire

16. Recherche menée par le Ministère des affaires municipales auprès de un maire sur cinq et de un conseiller sur vingt. 
entre 1981 et 1984 (tableau 1). Ces mairesses se trouvent surtout dans des petites municipalités, à l'exception des mairesses de deux villes de la Communauté urbaine de Québec, où l'une a été élue à l'occasion d'élections partielles à l'été 1985 (à Sillery) et l'autre a été élue à l'occasion des élections générales de novembre 1985 (à Sainte-Foy).

Si les élues ne sont pas plus nombreuses, le nombre des candidates semble pourtant s'être légèrement accru dans certaines

\section{TABLEAU 12}

Occupation des candidats élus, toutes les municipalités du Québec et villes de 20,000 habitants et plus.

\begin{tabular}{|c|c|c|c|c|}
\hline & $\begin{array}{l}1979 \\
\text { Maires et } \\
\text { conseillers } \\
\text { en poste }\end{array}$ & $\begin{array}{c}1981 \ldots \\
\text { Tous les } \\
\text { candidats élus } \\
\text { au poste de } \\
\text { maire }\end{array}$ & $\begin{array}{c}1983 \\
\text { Tous les } \\
\text { candidats élus } \\
\text { au poste de } \\
\text { maire }\end{array}$ & $\begin{array}{c}1978 \text { à } 1981 \\
\text { Tous les } \\
\text { candidats élus } \\
\text { dans les villes } \\
\begin{array}{c}20,000 \text { H. et } \\
+\end{array}\end{array}$ \\
\hline $\begin{array}{l}\text { Administrateur, banque, } \\
\text { assurance, industriel, } \\
\text { commerçant }\end{array}$ & $23 \%$ & $29 \%$ & $25 \%$ & $48 \%$ \\
\hline Agriculteur & $23 \%$ & $27 \%$ & $27 \%$ & $1 \%$ \\
\hline $\begin{array}{l}\text { Professionnel, } \\
\text { enseignant }\end{array}$ & $11 \%$ & $9 \%$ & $9 \%$ & $23 \%$ \\
\hline Salarié, travailleur & $20 \%$ & $24 \%$ & $25 \%$ & $25 \%$ \\
\hline $\begin{array}{l}\text { Autre } \\
\text { (ménagère, retraité, } \\
\text { étudiant, chômeur) }\end{array}$ & $23 \%$ & $10 \%$ & $12 \%$ & $4 \%$ \\
\hline $\mathbf{N}$ & ND & 873 & 903 & 455 \\
\hline
\end{tabular}

Source: Enquête du MAM auprès des élus municipaux, 1979. MAM, Direction de la recherche et des politiques, 1981 et 1983 . Colloque «Les principaux aspects de la réforme électorale au niveau municipal». Statistiques et analyse relatives à l'application des chapitres II, III, et VII de la Loi sur les élections dans certaines municipalités. Assemblée Nationale du Québec. 1982. 
municipalités. Par exemple, à Sainte-Foy, il y a neuf candidates sur un total de quarante-deux candidats en 1985; à Québec, trois candidates sur un total de quarante-six en 1981, et sept sur un total de quarante-cinq en 1985. Comme nouvelles venues sur la scène politique locale, les candidates ne sont pas aussi connues que leurs collègues de sexe masculin et ne bénéficient pas encore de l'avantage important au niveau municipal d'être une mairesse ou une conseillère sortant de charge.

L'utilisation de l'occupation des dirigeants municipaux jette un éclairage aussi intéressant sur la représentativité des élus. Dans l'ensemble du Québec, les élus municipaux se partagent presqu'également entre trois catégories: agriculteur, salarié et travailleur, et administrateur, industriel, commerçant (tableau 12). La quatrième catégorie en ordre d'importance, celle des professionnels et enseignants, est plus considérable dans les villes que dans les municipalités rurales, compte tenu que cette catégorie supérieure d'occupation est aussi plus largement représentée auprès des citoyens des villes. On constate par ailleurs un profil nettement différent dans les villes en ce qui concerne la catégorie «administrateur, commerçant, industriel» où sont regroupés $48 \%$ des candidats élus aux postes de maires et de conseillers entre 1978 et 1982. Presque la moitié des élus locaux proviennent donc du milieu des affaires et de la gestion et s'y identifient comme candidats. Par contre, la catégorie des salariés et travailleurs spécialisés et non-spécilisés occupe une place à peu près constante, dans les villes comme dans les campagnes. Ce groupe est loin d'occuper ici une place équivalente à celle qui est la sienne au sein de la population dont il représente les $70 \%$ en 1981 .

Une comparaison rapide avec la population permet de constater: que les femmes sont grandement sous-représentées, puisqu'elles constituent $51 \%$ de la population totale; que certaines catégories d'occupation comme administrateur, commerçant, industriel et professionnel y sont sur-représentées; que la catégorie 
ouvrier et salarié y est sous-représentée; et enfin que ce genre de répartition statistique ne permet pas de rejoindre les retraités, les chômeurs et les ménagères qui constituent au moins $20 \%$ de la population totale sans pour autant être pris en compte au sein de la population active ${ }^{17}$.

\section{La démocratie, après les élections}

Si la démocratie ne s'exerçait qu'à tous les quatre ans, il ne serait pas nécessaire de se demander ce qui se passe entre les périodes électorales. Mais puisque la démocratie de représentation réfere également, selon notre définition, aux droits et devoirs des élus de même qu'à ceux des citoyens, il importe de se pencher sur le mode de fonctionnement du législatif et de l'exécutif au niveau municipal.

Les conseils municipaux québécois comptent un nombre restreint d'élus, à l'exemple des municipalités américaines d'où elles tiennent cette tradition. Si ce principe peut sembler acceptable dans le cas des petites municipalités, il l'était moins dans les grandes villes et les dispositions législatives récentes augmentant le nombre de districts électoraux ont constitué un pas dans la bonne direction. Encore faut-il que cette quinzaine ou cette vingtaine d'élus aient un rôle à jouer dans la gouverne de la municipalité.

Le partage des tâches et la place réservée aux conseillers varient selon qu'il s'agisse d'une ville où il y a des partis politiques et où le législatif doit partager ses pouvoirs avec un comité exécutif. Dans ce cas, il arrive que les conseillers de l'opposition et même

17. Au recensement du Canada de 1981, (Catalogue 93-965, tableau 10) la population active du Québec se répartit comme suit :

$\begin{array}{lrr}\text { administrateurs, directeurs } & : & 9 \% \\ \text { professionnels, enseignants } & : & 16 \% \\ \text { salariés et ouvriers } & : & 70 \% \\ \text { agriculteurs, miniers, pêcheurs : } & 4 \% \\ \text { Autres } & : & 1 \%\end{array}$


ceux du parti majoritaire qui ne font pas partie de l'exécutif, ne soient membres d'aucun comité ou d'aucune commission hormis le conseil municipal qui se réunit généralement à toutes les deux semaines. On cite l'exemple du conseil municipal de la ville de Longueuil dont les dix-neuf conseillers réussissent à passer à travers un ordre du jour comptant une soixantaine de points, en quatrevingt dix minutes.

La pratique fort répandue des comités pléniers qui précèdent les assemblées publiques du conseil municipal, et où se tiennent les discussions et se réalisent les compromis, rend possible cette course contre la montre au conseil municipal, où les numéros de règlement et les résolutions se succèdent à un rythme accéléré.

Certains voient dans cette pratique une raison valable pour accroître l'intérêt pour les débats municipaux, notamment par le moyen, utilisé à d'autres niveaux, de la télédiffusion. Les séances du conseil municipal sont en effet télédiffusées dans quelques villes, comme à Longueuil et à Baie-Comeau ${ }^{18}$. La retransmission des débats imposerait une démarche de simplification et même de présentation de l'essentiel des questions débattues dont les conseillers «ordinaires» seraient les premiers bénéficiaires.

Une implication accrue des élus dans la gestion politique municipale exigerait plus de ressources et plus de temps de leur part. Pour la majorité d'entre eux, il s'agit d'une tâche qu'ils remplissent à temps partiel ${ }^{19}$, et avec peu de ressources de recherche et de secrétariat.

18. Selon le maire de Baie-Comeau, cette pratique serait efficace dans la situation particulière de cette ville où, suite au regroupement de 1982, les représentants des parties est et ouest de la ville pourraient avoir des échanges peu "parlementaires" si ce n'était de la télédiffusion des débats qui les incite à la modération (Le Soleil, 20 octobre 1985, p. A7, «Baie Comeau: nouveau géant de la Côte-Nord»).

19. Sur ce point, il y a quelques exceptions. Parmi les maires des grandes villes, plusieurs s'y consacrent à plein temps. À Montréal, dix des quatorze représentants du RCM au Conseil municipal réservent tout leur temps à cet «emploi» en 1985. 
En plus d'agir comme représentants des citoyens au conseil municipal, une partie des élus locaux ont aussi comme tâche de siéger au niveau régional, soit à la communauté urbaine ou régionale, soit à la Municipalité régionale de comté (MRC). Ceux qui n'y sont pas délégués, doivent nonobstant suivre les questions qui y sont discutées et appuyer leurs représentants. On constate dans la pratique que les élus locaux ne s'intéressent au niveau régional que très peu, qu'ils acceptent mal d'être questionnés à ce propos aux séances de leur conseil municipal, et que, par ailleurs, ils ont peu de possibilités d'être plus étroitement associés aux discussions régionales. Les comités et commissions qui pourraient les y inciter sont en effet peu nombreux et de formation récente. Il arrive aussi que les représentants au conseil des partis minoritaires soient totalement exclus de la délégation de la ville au niveau régional ${ }^{20}$, ce qui a pour conséquence de rendre difficile sinon d'empêcher toute intervention de l'opposition sur des questions comme l'aménagement du terrritoire ou le transport en commun.

Les élus municipaux ne constituent donc pas un bloc homogène, tant par leur profil que par la place qu'ils occupent dans la gouverne locale. Certains d'entre eux bénéficient d'un statut spécifique au sein de l'appareil municipal (par exemple le maire, les membres du comité exécutif, les représentants du parti majoritaire) qui leur vaut une place privilégiée, ce qui contrevient au principe de l'égalité des élus par rapport à l'information et au pouvoir.

\section{La démocratie de participation}

Une partie importante des discussions portant sur le gouvernement local concerne la place de la démocratie de participation.

20. C'est le cas à Québec où les quatre représentants du RPQ n'ont pas siégé au conseil de la CUQ entre 1981 et 1985. 
Parmi les expériences d'autogestion, plusieurs ont en effet lieu dans le champ du gouvernement municipal de sorte qu'il devient pertinent de se pencher sur ces pratiques et d'en évaluer la place par rapport à la scène politique et à la société locales.

En principe, la démocratie de participation signifie que les gens concernés par une décision sont également ceux qui la prennent. Puisqu'il a été constaté que, dans les municipalités québécoises, la démocratie de représentation était le modèle dominant, la participation doit donc surtout se réaliser en marge ou en parallèle avec la délégation de pouvoir que les citoyens font à leurs dirigeants politiques municipaux.

Tout comme dans la section précédente, la situation ici doit être analysée en fonction du milieu. La question de la participation ne se pose pas, en effet, selon les mêmes termes dans un village de mille habitants où les élus et les citoyens se côtoient quotidiennement, et dans une ville de 50,000 habitants. Les premières revendications populaires au sujet de la participation ont d'ailleurs été articulées dans les grandes villes, à la fin des années soixante.

\section{Les revendications participationnistes}

Cette période ne fut pas le fait du hasard, mais elle montre, bien au contraire, comment doit être compris le phénomène de la participation. Au cours des années soixante en effet, les centres urbains québécois connaissent des modifications profondes: rénovation urbaine, modernisation du réseau routier, implantation au centre-ville des sièges sociaux des grandes entreprises, démolition et relocalisation partielle, etc. Écartés de ces décisions qui les touchent pourtant de près, les résidents se regroupent et revendiquent une participation aux processus décisionnels tout en faisant connaître de différentes façons leurs points de vue.

Les mécanismes institutionnels de consultation et de participation sont peu nombreux et ne satisfont pas les citoyens. 
Associés à quelques comités où ils sont minoritaires, aux côtés de représentants de d'autres milieux tels les milieux d'affaires et les milieux des spécialistes, les citoyens constatent que leurs intérêts n'y sont pas pris en compte et que ces instances ne sont que consultatives.

De plus, les citoyens connaissent eux aussi des problèmes de représentativité (EZOP-Québec, 1981, 371). À peine émancipés de l'influence du clergé paroissial, particulièrement dans les quartiers défavorisés des grandes villes, les groupes de citoyens ressentent lourdement la présence des intellectuels et des professionnels de l'animation (Hamel, 1983, 77). De plus, leur base de mobilisation étant en général découpée en fonction des limites, les quartiers, le pouvoir municipal les identifie à des intérêts sectoriels marqués par «l'esprit de clocher». La difficulté qu'ils éprouvent à se regrouper et à faire front commun accorde une certaine crédibilité à cet argument, auquel s'ajoute peu à peu un ralentissement de la mobilisation à la base, du moins en ce qui concerne la participation institutionnalisée.

Au cours des années soixante-dix, les groupes populaires ont tenté d'éviter le déplacement des enjeux vers des enjeux de plus en plus partiels. Mais leurs efforts d'interventions reliées au développement économique et à l'aménagement se sont avérés très difficiles et ont connu peu de succès, notamment pour deux raisons. D'abord, à cause de l'encadrement institutionnel de leur participation, aux programmes de rénovation et d'amélioration de quartier par exemple, où leurs interventions étaient restreintes aux petits équipements plutôt qu’à l'aménagement du quartier (Charbonneau, 1985). En deuxième lieu, les difficultés de mobilisation de la base, l'essouflement des militants de la première heure et la lenteur de leur remplacement par d'autres, ont créé des conditions qui s'accomodaient bien de questions plus «étroites", plus susceptibles d'intéresser les «usagers» et les résidents des quartiers (Martin, 1985). 


\section{La participation institutionnalisée: le référendum}

Parmi les formes de participation institutionnalisée, le référendum peut relever du modèle de la démocratie représentative comme de celui de la démocratie participative, selon la place qui lui est accordée dans le processus décisionnel. Formellement, le référendum tel qu'il peut être tenu dans les municipalités québécoises, a une portée consultative seulement et sert à guider la décision des élus municipaux. En pratique, les dirigeants locaux se sentent liés politiquement par les résultats d'une telle consultation, à laquelle ils ont d'ailleurs parfois recours pour consolider leur propre position face aux gouvernements supérieurs (comme dans le cas des référendums portant sur des projets d'annexion de territoire ${ }^{21}$ ) ou pour se dégager d'une décision difficile à prendre (comme dans le cas des référendums portant sur les amendements au règlement de zonage où les élus sont "pris» entre les droits acquis des résidents et les intérêts des promoteurs).

Sans qu'il s'agisse d'une pratique courante, le référendum existe au niveau municipal, alors qu'il est très exceptionnellement utilisé aux autres niveaux politiques. Traditionnellement, le référendum a été l'occasion d'abolir la prohibition de la vente des boissons alcooliques dans les municipalités québécoises entre 1945 et 1955. Il était aussi souvent utilisé par les élus locaux qui réussissaient ainsi à faire accepter une augmentation de leurs émoluements ${ }^{22}$.

La possibilité de tenir une consultation populaire est aujourd'hui surtout réservée aux amendements au règlement de

21. Dans la suite de la "Loi favorisant le regroupement » adoptée par l'Assemblée Nationale du Québec en 1971 , le référendum peut être tenu dans le cas de projets de regroupements municipaux. De tels référendums ont eu lieu à Sillery et à Loretville en 1973 et les résidents se sont opposés aux projets.

22. Cette pratique n'est plus nécessaire maintenant que les élus municipaux reçoivent une rémunération assez substantielle dont les bases minimales sont fixées par la loi. 
zonage et aux règlements d'emprunt. Dans le premier cas, les personnes habilitées à voter sont en général peu nombreuses, puisque la consultation se limite aux «zones» immédiatement touchées par l'amendement projeté.

Si la possibilité juridiquement reconnue de consulter les citoyens existe, les référendums sont rarement tenus. En fait, l'organisation elle-même d'une telle consultation effraie les responsables municipaux, d'autant plus que les dispositions relatives au financement des dépenses électorales ne s'appliquent pas ici.

Les résidents ne partagent pas le même point de vue et sont plutôt favorables à la tenue de référendum. Dans les trois plus grandes villes du Québec (Montréal, Laval et Québec) où la possibilité de tenir une telle consultation populaire n'existe pas, les forces d'opposition en ont fait l'objet de revendications répétées depuis une dizaine d'années, notamment en ce qui concerne le zonage et l'aménagement.

Les quelques cas de référendums municipaux tenus au Québec indiquent que l'intérêt des résidents se manifeste davantage pour les projets de zonage (qui touchent immédiatement les droits acquis des propriétaires et le milieu de vie) que lorsqu'il s'agit de projets d'emprunts. La question fiscale a cependant gagné en importance au cours des dernières années alors que le financement des projets municipaux par emprunts se faisait plus facilement il y a dix ans.

L'intérêt du référendum ne tient pas strictement aux quelques applications qui en ont été réalisées, mais plutôt à la possibilité qu'il offre aux résidents de se mobiliser et de manifester leur intention de s'opposer à un projet. Le référendum est ainsi surtout une «menace» puisque le fait de réunir un nombre minimal de signature de résidents opposés à un projet et demandant la tenue du référendum suffit dans la plupart des cas à retarder, à modifier ou à retirer le projet. 
Les différentes modalités de participation des citoyens à la gouverne de la société locale ne semblent pas remettre en question la place qui est réservée aux élus municipaux. Ces derniers entretiennent un réseau de consultation où l'ensemble des résidents n'occupent pas tout l'espace ni même une portion importante de cet espace. Parmi les avenues proposées comme façon de remédier à cette situation en vue de remettre entre les mains des résidents une certaine part des décisions qui les concernent, la formule du conseil de quartier a été pronée par les groupes populaires et certains partis municipaux.

\section{Les conseils de quartier}

Encore inexistante en pratique dans les municipalités québécoises, la formule du conseil de quartier impliquerait une délégation de pouvoir au niveau infra-municipal. Le principe est surtout valable dans les grandes villes où la gestion centralisée de services comme par exemple ceux des loisirs, de la circulation, ou de l'urbanisme, rend difficile la considération des spécificités des quartiers.

Les bureaux de quartier ouverts depuis quelques années dans certaines villes ne répondent pas aux mêmes besoins puisqu'ils visent à informer les résidents. Par les conseils de quartier, des représentants des résidents se verraient confier un pouvoir, consultatif ou décisionnel selon le modèle retenu, dans un ensemble plus ou moins large de domaine, sous l'autorité et la coordination du conseil municipal. Déjà implantés aux États-Unis et dans certains pays scandinaves et européens, les conseils de quartier sont vus dans les villes américaines comme un moyen de permettre aux communautés ethniques d'exercer un certain contrôle sur leur milieu de vie, et dans les villes norvégiennes et italiennes comme une façon de rapprocher les citoyens des services sociaux dont le niveau municipal a la charge (Dente, 1980; Kjellberg, 1981). 
Présentés par le RCM à Montréal, et par le RPQ à Québec, comme une condition essentielle à la démocratisation des décisions municipales, les conseils de quartier exigeraient une volonté d'implication des citoyens au niveau des quartiers et une attitude de collaboration de la part de l'administration municipale où l'information est présentement centralisée ${ }^{23}$.

\section{Les carences de la démocratie locale}

En examinant les voies institutionnelles de la représentation et de la participation dans les municipalités québécoises, nous constatons qu'il n'y a pas lieu de conclure en l'existence d'une gouverne politique respectueuse de tous les critères démocratiques. Ainsi, dans plus de la moitié des municipalités, les dirigeants ne sont pas choisis par scrutin mais désignés par acclamation. Là où il y a contestation des postes et scrutin, la participation électorale est en général plus faible qu'aux autres niveaux politiques. Les dirigants élus ont un profil qui ne correspond pas à celui de la population. Enfin une bonne partie des décisions échappe à l'ensemble des dirigeants municipaux. Ces constatations remettent en cause les principes de légitimité, d'égalité et de respect de la minorité qui devraient caractériser les rapports démocratiques entre les élus et les citoyens. Il existe cependant des exemples de sociétés où certaines inégalités sont en voie d'être réduites. Ainsi, par exemple, les auteures d'une étude sur la participation des femmes dans cinq pays du nord de l'Europe constatent-elles que $29 \%$ des postes aux conseils municipaux en Suède sont occupés par des femmes. De plus, cette proposition est passée de $25 \%$ en

23. La formule initialement proposée par les deux partis a subi une évolution importante au cours des dernières années. Les chefs de parti en témoignent eux-mêmes (La Presse, 3 juin 1985, p. A2). 
1970 à $47 \%$ en 1982 au conseil municipal de Stockholm (HaavioMannila et al., 1985, 184-185). En Norvège, l'impact de procédures de désignation des représentants au conseil régional apparaît clairement alors qu'en 1971, 5\% des délégués des municipalités aux conseils régionaux sont des femmes nommées par l'ensemble des élus municipaux. Lorsque ces représentants sont élus directement, en 1975 , le pourcentage des femmes passe à $25 \%$, puis à $33 \%$ en 1983. (Haavio-Mannila, et al. 1985,84). Parmi les facteurs explicatifs de cette représentation exceptionnelle des femmes, par comparaison à la situation observée dans d'autres pays européens et nord-américains, les auteures mentionnent les caractéristiques socio-économiques (les revenus et le niveau de scolarité des hommes et des femmes étant plus rapprochés que dans d'autres pays), culturelles et politiques (l'État-providence est très présent et les lignes partisanes existantes au niveau central se retrouvent avec autant de force au niveau local) et idéologiques (la définition du champ politique, du public et du privé est spécifique à ces pays).

Cet exemple suggère qu'une voie de sortie de ce que certains appellent la crise de la démocratie pourrait se trouver du côté des réformes institutionnelles. C'est la voie adoptée par le Gouvernement du Québec avec son projet de valorisation du pouvoir local et de décentralisation ${ }^{24}$. Dans la même veine, il y aurait lieu de modifier le mode de désignation du maire, d'assurer le respect des droits de la minorité parlementaire et adopter des mesures susceptibles d'augmenter l'intérêt des citoyens pour les questions municipales (par exemple que les élections municipales soient tenues la même année pour toutes les municipalités). Outre une amélioration des procédures électorales, le transfert au niveau régional ou municipal de certaines responsabilités, comme le laisse entrevoir le projet

24. Ce projet touche la fiscalité municipale, la démocratie locale et l'aménagement du territoire. 
de décentralisation actuel, pourrait contribuer à rendre plus fondamentaux les enjeux locaux. Toutefois, la portée exacte du projet de décentralisation est difficile à cerner, puisque la décentralisation n'est pas en soi synonyme de démocratie et que ces deux termes pourraient même s'opposer (Langrod, 1981, 6-8).

Par ailleurs, les carences de la représentation pourraientelles être palliées par une valorisation des principes de la démocratie de participation? L'engagement communautaire, comme deuxième voie de sortie de la crise de la démocratie, a déjà été expérimenté. Ainsi des groupes de citoyens et des associations de résidents ont décidé et réussi jusqu'à un certain point à remettre la responsabilité de questions proches de la vie quotidienne des gens entre les mains d'organisations communautaires reconnues et supportées par la municipalité. Il leur a d'abord fallu vaincre les fractions adverses, particulièrement celles qui sont reliées aux intérêts immobiliers, et élire une majorité de leurs représentants au conseil municipal (Shearer, 1984, 583-585). Dans cette optique, la décentralisation et la responsabilisation des citoyens, qui sont souvent présentées comme des solutions aux problèmes de la représentation au niveau municipal, demeurent pour certains une formule administrative insatisfaisante. La solution se trouverait plutôt du côté de l'élargissement du débat politique et d'un accroissement des moyens d'intervention des citoyens (Bassand et Fragnière, 1978, 179).

Une troisième voie de solution se trouve du côté d'un repli sur la sphère du privé. Elle impose une rupture avec la définition traditionnelle du champ politique pour rejoindre ceux et celles qui affirment que la démocratie passe aussi par la sphère de la vie domestique ou privée (Haavio-Mannila et al., 1985, XVI). Plusieurs arguments sont présentés à l'appui de cette thèse: dévalorisation et perte de sens du suffrage universel, abandon de la notion d'État-providence, rétrécissement du champ traditionnel 
de l'intervention de l'État. Le repli sur le quotidien, le court terme, les nouveaux modes de vie, «le combat sur les terrains les plus proches, là où sont encore visibles, par tous, les enjeux réels... voilà des attitudes authentiquement politiques, bien à tort dévaluées par la professionnalisation de la politique» (Braud, 1980, 244).

La façon dont est mené le débat sur la démocratie locale au Québec actuellement présente plusieurs points communs avec la remise en question de la place des pouvoirs locaux dans d'autres pays. C'est un débat qui reste ouvert et les enjeux qui le soustendent concernent la société et l'agencement politique des rapports sociaux.

\section{Bibliographie}

ANDREW, Caroline. «Les femmes et la consommation collective: les enjeux de l'engagement politique». Politique, vol. 5 (hiver 1984), 109-122.

BASSAND, Michel, Jean-Pierre FRAGNIÈRE. Le pouvoir dans la ville, Vevey, Éditions Delta, S.A., 1978, 221 p.

BEAUREGARD, Ludger. "Les élections municipales à Montréal en 1982: une étude de géographie politique». Cabiers de géographie du Québec, vol. 28, no. 75 (décembre 1984): 395-433.

BERNARD, André. La politique au Canada et au Québer. Montréal, Les Presses de l'Université du Québec, 1976. 516 p.

BRAUD, Philippe. Le suffrage universel contre la démocratie. Paris, Presses universitaires de France, 1980. 246 p.

CASTELLS, Manuel. La ville, la démocratie, le socialisme. Bruxelles, Contradictions, 1982. $192 \mathrm{p}$.

CHARBONNEAU, Johanne. Partis politiques et enjeux urbains: évaluation de la démocratie municipale à Québec: 1966 - 1984. Thèse en cours en vue d'obtenir le grade de maitre en Aménagement du territoire et développement régional, Université Laval, 1985.

DENTE, Bruno, Gloria REGONINI. «Urban policy and Political Legitimation: The Case of Italian Neighborhood Councils». International Political Science Review, Vol. no. 2 (1980): 187-202.

EZOP-QUÉBEC. Une ville à vendre. Laval, Éditions coopératives Albert Saint-Martin, 1981 (original 1972), $559 \mathrm{p}$. 
FORAND, Claude. Problèmes urbains et participation électorale à Sainte-Foy: 1969 à 1977. Thèse présentée en vue de l'obtention du grade de maitre ès Arts (Science politique), Université Laval, 1985.

GODBOUT, Jacques. La participation contre la démocratie. Montréal, Éditions coopératives Albert Saint-Martin, 1983, 190 p.

HAAVIO-MANNILA, Elina et al. Unfinished Democracy Women in Nordic Politics. Oxford, Pergamon Press, 1985, 206 p.

HAMEL, Pierre. Logement et luttes urbaines à Montréal. Cahier de recherche. Montréal, Faculté de l'aménagement, Université de Montréal, 1983, 321 p.

HAMEL, Pierre, Jean-François LEONARD. Les organisations populaires, l'État et la démocratie. Montréal, Nouvelle optique, 1981, 208 p.

HIGGINS, Donald J.H. Urban Canada: Its Government and Politics. Toronto, The Macmillan Company of Canada Ltd, 1977. 322 p.

JOYCE, J.G., H.A. HOSSÉ. Civic Parties in Canada. Ottawa, Fédération canadienne des maires et des municipalités, 1970, 113 p.

KJELLBERG, Francesco. "Two Models of Municipal Decentralization: Bologna and Oslo». Paper prepared for the European Meeting on Applied Urban Research, Essen, 1981, $32 \mathrm{p}$.

KOLM, Serge-Christophe. Les élections sont-elles la démocratie? Paris, Les Éditions du Cerf, $1977,138 \mathrm{p}$.

LANGROD, Georges. "Local Government and Democracy», Lionel D. Feldman (Ed.). Politics and Government of Urban Canada (4th Edition). Toronto, Methuen, 1981 (Original 1953). p. 3-14.

LEVEILLE, Jacques. «Les alliances politiques à Montréal : réaménagement ou mutation? Possibles, vol. 3, no. 1 (automne 1978): 75-94.

LIJPHART, Arend. Democracies: Patterns of Majoritarian and Consensus Government in TwentyOne Countries. New Haven, Yale University Press, 1984, 229 p.

MACPHERSON, C.B. Le véritable monde de la démocratie (traduit de l'anglais par C. Dufresne). Montréal, Les Presses de l'Université du Québec, 1976 (Original 1966). 75 p.

MAGNUSSON, Warren, Andrew SANCTON. City Politics in Canada. Toronto, University of Toronto Press, 1983. $338 \mathrm{p}$.

MARTIN, Josée. Les groupes de quartier à Québec: luttes et contraintes politicn-institutionnelles. Université Laval. Les Cahiers du CRAD vol. 9, no. 4, 1985. 117 p.

MILLS, C. Wright. L'imagination sociologique. Paris, Fançois Maspéro, 1977 (Original 1967). 229 p.

QUESNEL-OUELLET, Louise. "Les partis politiques locaux au Québec», V. Lemieux (dir.). Personnel et partis politiques au Québec. Montréal, Boréal Express, 1982. p. 277-306.

SHEARER, Derek. "Citizen Participation in Local Government». International Journal of Urban and Regional Research, vol. 8, no. 4 (1984): 573-586.

SAINT-PIERRE, Marc. "Baie Comeau: nouveau géant de la Côte-Nord». Le Soleil, 20 octobre 1985 , p. A7.

TINDAL, C.R., S. Nobes TINDAL. Local Government in Canada (Second Edition). Toronto, McGraw Hill Ryerson Ltd, 1984. 272 p.

VACHET, André. "La participation contre la démocratie». Recensions, Politique: (Hiver 1985) no. $7: 165-170$. 